\title{
AVALIAÇÃO DA CONDIÇÃO DE SAÚDE BUCAL DE PACIENTES EM PROGRAMA DE TRANSPLANTE DE ÓRGÃOS SÓLIDOS
}

\author{
Assessment of oral health status of patients on program for solid organ transplant
}

\author{
Catharine Bittencourt Cunha', Erika Christina Amantes de Souza', Perla Porto Leite Shitara', Paulo Sérgio da Silva Santos², \\ Luciene Luvizotto David'.
}

\begin{abstract}
RESUMO
Pacientes portadores de doenças crônicas em programa de transplante de órgãos sólidos apresentam condições sistêmicas que comprometem seu sistema imune, o que os predispõe a risco potencial de infecções, que devem ser prevenidas para o momento pós-transplante, principalmente diante do uso de imunossupressores. Os focos infecciosos em cavidade oral após o transplante são uma preocupação com essa condição e merecem atenção especial. Objetivo: Avaliar dados referentes à condição da saúde bucal de pacientes em programa de transplante de órgãos sólidos, buscando evidenciar a importância da assistência odontológica prévia. Métodos: Este estudo teve caráter quantitativo, descritivo, exploratório e retrospectivo, onde foram analisados 225 prontuários de pacientes em programa de transplante de fígado, rim e coração, os dados referentes à condição bucal e os possíveis riscos infecciosos (cáries, doença periodontal e lesões infecciosas de mucosas) obtidos nos relatórios de avaliação odontológica. Foram aplicados métodos estatísticos para análise de relevância através do teste de qui-quadrado e teste exato de Fisher com significância $(p<0,05)$. Resultados: Foi registrada a presença de alterações bucais sinalizadoras de focos de infecção em (134/166) 80,72\% pacientes em programa de transplante hepático, (35/43) 81,39\% em programa de transplante renal e (13/16) 81,25\% de transplante cardíaco. Conclusão: Os dados obtidos permitiram concluir que nos pacientes em programa de transplantes de órgãos, a incidência de focos infecciosos bucais é significativa e sugerem que a adequação bucal prévia aos transplantes é relevante diante da condição de imunossupressão a que aqueles pacientes são submetidos.
\end{abstract}

Descritores: Transplante de fígado; Transplante de rim; Transplante de Coração; Infecção; Saúde bucal; Prevenção \& Controle.

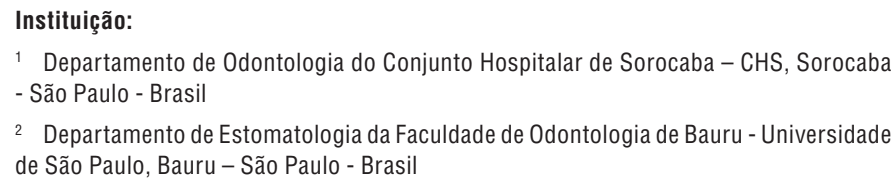

1 Departamento de Odontologia do Conjunto Hospitalar de Sorocaba - CHS, Sorocaba - São Paulo - Brasil

2 Departamento de Estomatologia da Faculdade de Odontologia de Bauru - Universidade de São Paulo, Bauru - São Paulo - Brasil

\section{Correspondência:}

Dra. Catharine Bittencourt Cunha

Avenida Sete de Setembro, 2847, AP 1401- A - Cep 40130-000 Salvador-BA.

Tel: (71) 9987-0592

Email: kk_bittencourt@ig.com.br

\section{INTRODUÇÃO}

O transplante de órgãos é aceito como modalidade terapêutica efetiva nas doenças graves avançadas e seu sucesso depende de uma completa infra-estrutura hospitalar e de uma equipe multiprofissional altamente treinada no procedimento e no acompanhamento de pacientes gravemente debilitados e imunodeprimidos. ${ }^{1}$ A imunossupressão medicamentosa é obrigatória em todos os casos, mesmo naqueles em que a imunidade já está gravemente comprometida pela doença e o esquema tríplice de imunossupressão baseia-se em corticosteróides, ciclosporina e azatioprina, método adotado em diferentes centros. ${ }^{2}$ Demais drogas imunossupressoras sugeridas na literatura, tais como os antibióticos macrolídeos (Tracolimo e Sirolimo). O Tracolimo é uma estratégia efetiva a ser aplicada em pacientes que apresentam rejeição refratária após terapia baseada em ciclosporina, enquanto o sirolimo é utilizado em rejeições crônicas. ${ }^{3}$ A dose selecionada deve prevenir a rejeição e a infecção do órgão transplantado, que podem ser extremamente graves, especialmente no pós-operatório imediato. A causa de óbito mais frequente é a infecção tanto no pós-operatório imediato como no tardio. ${ }^{4} \mathrm{~A}$ cavidade oral é um ecossistema aberto que permite a entrada e saída de várias espécies de microrganismos durante toda a vida do hospedeiro. É composto de vários nichos e possui uma microbiota nativa bastante complexa, conforme exemplificado na Figura $1 .{ }^{5}$ As composições quantitativa e qualitativa da microbiota oral está diretamente relacionada a fatores reguladores que podem propiciar o crescimento ou a inibição da microbiota. ${ }^{6}$ 
O enigma da boca consiste da simbiose natural e simultaneamente das infecções endógenas, que culminam com a quebra do equilíbrio existente na cavidade oral. ${ }^{4,6}$ A microflora que protege pode ao mesmo tempo causar doenças locais e sistêmicas. A erupção dentária na cavidade oral leva imediatamente à colonização desta por microorganismos, o que irá formar o biofilme e, consequentemente, a placa dental, a qual é responsável pela formação da cárie dental. ${ }^{7}$

Figura 1: Microrganismos relacionados à cárie dental

\begin{tabular}{|c|c|c|c|}
\hline $\begin{array}{l}\text { - Streptococcus } \\
\text { mutans }\end{array}$ & $\begin{array}{l}\text { - Porpyromonas } \\
\text { endodontalis }\end{array}$ & $\begin{array}{l}\text { - Campylobacter } \\
\text { gracilis }\end{array}$ & $\begin{array}{l}\text { Bacterioides } \\
\text { species }\end{array}$ \\
\hline $\begin{array}{l}\text { - Streptococcus } \\
\text { sobrinus }\end{array}$ & $\begin{array}{l}\text { - Eubacterium } \\
\text { species }\end{array}$ & $\begin{array}{l}\text { - Leptotrichia } \\
\text { bucallis }\end{array}$ & $\begin{array}{l}\text { - Fusobacteeerium } \\
\text { nucleatum } \\
\text { sp.vicenti, }\end{array}$ \\
\hline - Lactobacillus & $\begin{array}{l}\text { - Fusobacterium } \\
\text { nucleatum }\end{array}$ & - Neisseria & - Lactobacillus \\
\hline - Actinomyces & $\begin{array}{l}\text { - Peptostreptococcus } \\
\text { micros }\end{array}$ & & - Actinomyces \\
\hline
\end{tabular}

Fonte: Gill Y, Scully C. Orofacial odontogenic infections: review of microbiology and current treatment. Oral Méd Oral Pathol 1990;70:156.

A progressão da doença cárie promove o envolvimento da polpa dental, e sua exposição resulta em desequilíbrio dessa flora simbiótica natural, provocando necrose pulpar e abscesso alveolar, podendo comprometer sistemicamente o hospedeiro. ${ }^{6-9,0}$

Indivíduos que escovam mais os dentes têm menos placa, o que consequentemente diminui as afecções sistêmicas. ${ }^{10}$ Por isso a necessidade da orientação com relação à higienização e adequação da cavidade oral na prevenção de doenças sistêmicas infecciosas, principalmente quando o paciente encontra-se imunossuprimido. , $11^{2}$

O efeito colateral mais significativo nos tecidos periodontais é a hiperplasia gengival, que pode manifestar-se com várias intensidades, sempre na dependência de outros fatores como a placa bacteriana, que potencializa o efeito danoso dessa hiperplasia. ${ }^{12,13}$ O efeito combinado, ou seja, inflamação mais hiperplasia é um fato comprovado pela literatura, pela dificuldade do indivíduo em realizar uma higiene bucal eficiente. ${ }^{14-17}$

\section{MÉTODOS}

Este estudo transversal de caráter quantitativo, descritivo, exploratório e retrospectivo foi desenvolvido no Conjunto Hospitalar de Sorocaba-SP, tendo como população de estudo os pacientes portadores de disfunções hepáticas, renais e cardíacas graves listados para transplante de fígado, rim e coração, respectivamente, e que receberam avaliação odontológica prévia ao transplante através dos dados obtidos a partir dos prontuários hospitalares no período de 2002 a 2007.

O levantamento de dados foi realizado por meio de uma ficha criada especificamente para a pesquisa, que norteou a coleta de informações referentes à saúde sistêmica e bucal daqueles pacientes em seus respectivos prontuários médico/odontológicos, abrangendo informações sobre a presença de lesões cariosas e doença periodontal, focos infecciosos, lesões intra-ósseas em cavidade oral utilizando variáveis quantitativas. As informações sobre uso de próteses móveis e hábitos como etilismo e tabagismo também foram coletadas na amostra estudada.

Foram excluídos da amostra prontuários que apresentaram dados incompletos da avaliação odontológica, tornando-os não conclusivos e dados ilegíveis.

Todos os prontuários avaliados continham o Termo de Consentimento Livre e Esclarecido assinado no momento da internação pelo próprio paciente ou pelo responsável e o estudo foi submetido e aprovado ao comitê de Ética e Pesquisa do Conjunto Hospitalar de Sorocaba - Hospital Santa Lucinda.

Para análise dos resultados foram aplicados o Teste $\mathrm{G}$ de Cochran para comparação da frequência da alteração bucal encontrada separadamente nos sexos masculino e feminino; Teste do Quiquadrado para comparação dos diferentes grupos de transplantes de órgãos, frequência das alterações bucais, hábitos dos pacientes e o Teste de Fisher aplicado para comparar a incidência das alterações bucais para cada sexo, considerando o nível de significância de $5 \%(p=0,05)$.

\section{RESULTADOS}

A amostra totalizou 225 prontuários de pacientes listados para transplante hepático, renal e cardíaco de ampla faixa etária, de ambos os sexos, sendo $166(73,7 \%)$ prontuários listados para transplante hepático, $43(19,1 \%)$ para transplante renal e $16(7,2 \%)$ para transplante cardíaco.

As alterações bucais presentes nesse grupo de pacientes seguiu os seguintes critérios:

- Pelo menos uma lesão cariosa dentária visível clinicamente.

- Presença de qualquer grau de doença periodontal, gengivite e/ou periodontite por avaliação clínica visual ou através de sondagem periodontal.

- Lesões bucais de caráter infeccioso (fúngica, viral ou bacteriana)

Dentre os pacientes listados para transplante de fígado (133), $80,72 \%$ apresentaram alterações bucais. Dos pacientes listados para transplante renal (35), 81,40\% apresentaram problemas estomatológicos e dos pacientes listados para transplante cardíaco (13), 81,25\% apresentaram comprometimentos na boca. Esses dados estão exemplificados na Figura 2.

Figura 2: Relação de pacientes submetidos a transplantes de órgãos sólidos e apresentaram alterações estomatológicas no período de 2002 a 2007.

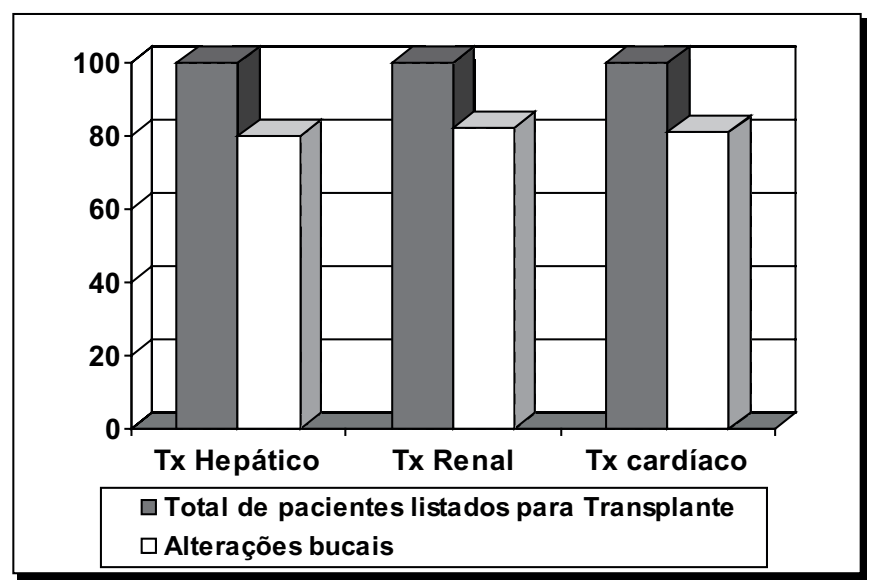


Tabela 1. Caracterização da doença cárie presente em pacientes submetidos a transplante hepático, cardíaco e renal.

\begin{tabular}{|c|c|c|c|c|c|c|c|}
\hline & Feminino & & & Masc & & & \\
\hline Transplante & Com & Sem & \%total & Com & sem & \%total & Fem $\times$ Masc \\
\hline Renal & 10 & 11 & $47,61 \%$ & 9 & 12 & $42,85 \%$ & $X^{2}=0,10$ \\
\hline Cardíaco & 6 & 2 & $75 \%$ & 3 & 5 & $37,5 \%$ & $P=0,1573 N S$ \\
\hline Hepático & 20 & 34 & $37,03 \%$ & 70 & 40 & $63,63 \%$ & $X^{2}=10,35(P<0,001)$ \\
\hline Total & 36 & 47 & $16 \%$ & 82 & 57 & $36,4 \%$ & \\
\hline
\end{tabular}

Em relação à doença cárie, o percentual dessa entidade patológica em relação ao tipo de transplante submetido foi dividido por gênero como é evidenciado na tabela 1.

Como podemos observar na Tabela 1 , os índices de cárie foram maiores no grupo de pacientes submetidos a transplante cardíaco (56,25\% dos casos) comparados aos transplantados hepáticos $(54,2 \%)$ e transplantados renais $(42,2 \%)$, independente do gênero. Tanto para o gênero feminino quanto para o masculino, o teste do qui-quadrado não mostrou diferença significante entre os transplantados de rim, fígado ou coração quando comparados em relação à presença de cárie. As mulheres que seriam submetidas a transplante cardíaco apresentaram índices de cárie mais elevados entre os grupos e gêneros (75\% dos casos). Ao comparar os dois gêneros, o teste mostrou para os transplantados de fígado que a percentagem de cárie no gênero masculino $(63,6 \%)$ foi significantemente maior do que a observada no gênero feminino (37,0\%). A análise mostrou associação significante entre a presença de cárie e o gênero masculino apenas nos transplantados de fígado.

Figura 3: Relação de pacientes submetidos a transplantes de órgãos sólidos divididos por gênero que apresentaram doença cárie no período de 2002 a 2007.

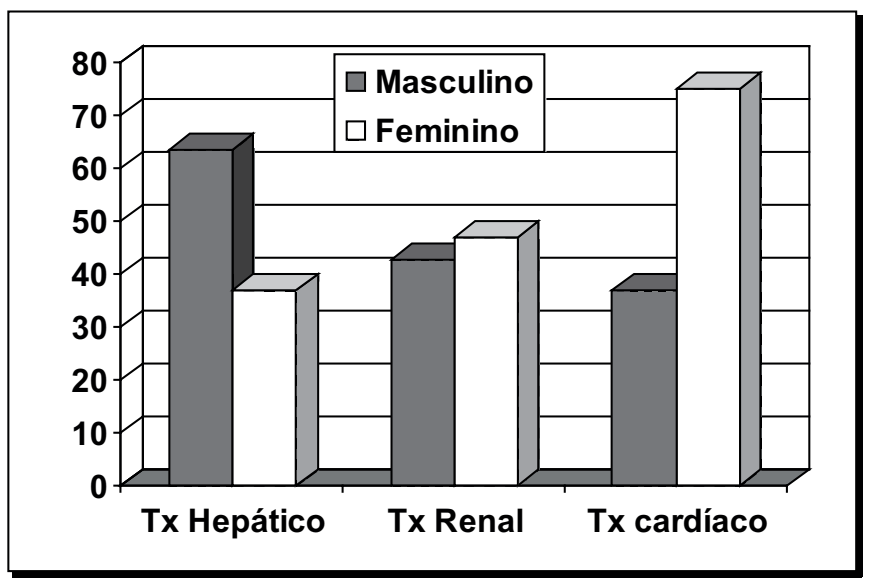

A Figura 3 exemplifica que os índices de cárie foram elevados entre os grupos, com uma percentagem maior no gênero feminino submetido a transplante cardíaco.

Em relação à doença periodontal, o percentual dessa entidade patológica em relação ao tipo de transplante submetido foi dividido por gênero, como é evidenciado na Tabela 2.

Em relação à doença periodontal, observou-se apenas no gênero feminino que os transplantados de rim ou coração apresentam percentagens de doenças periodontais $(66,7 \%$ e $87,5 \%$,respectivamente) significantemente maiores do que os transplantados hepáticos. Ao comparar os gêneros feminino e masculino, apenas no grupo do fígado a percentagem de homens (70,9\%) foi significantemente maior do que a apresentada pelo gênero feminino ( $38,9 \%$ ).

A Figura 4 exemplifica que os índices de doença periodontal foram elevados entre os grupos, com uma percentagem maior no gênero feminino submetido ao transplante cardíaco.

Figura 4: Relação de pacientes submetidos a transplantes de órgãos sólidos divididos por gênero que apresentaram doença periodontal no Conjunto Hospitalar de Sorocaba no período de 2002 a 2007.

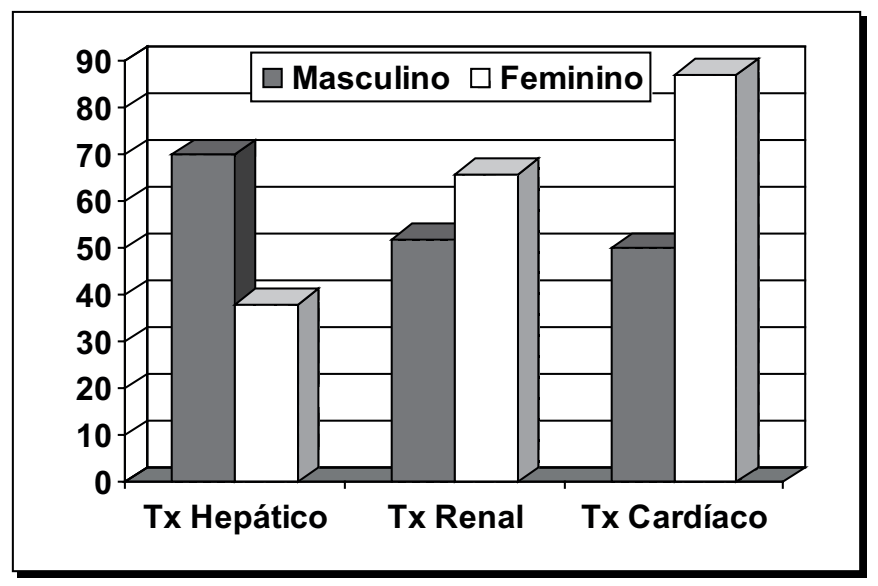

Tabela 2. Caracterização da doença periodontal presente em pacientes submetidos á transplante hepático, cardíaco e renal no Conjunto Hospitalar

\begin{tabular}{llllllll}
\hline & Feminino & \multicolumn{5}{c}{ Masculino } \\
\hline Transplante & Com & Sem & \%total & Com & sem & $\%$ total & Fem $\times$ Masc \\
Renal & 14 & 7 & $66,66 \%$ & 11 & 10 & $52,38 \%$ & $\mathrm{X}^{2}=0,89 \mathrm{NS}$ \\
Cardíaco & 7 & 1 & $87,5 \%$ & 4 & 4 & $50 \%$ & $\mathrm{P}=0,1410$ \\
Hepático & 21 & 33 & $38,88 \%$ & 78 & 32 & $70,09 \%$ & $\mathrm{X}^{2}=15,52(\mathrm{P}<0,001)$ \\
Total & 42 & 41 & & 93 & 46 & & \\
\hline
\end{tabular}


Na Tabela 3 são abordados os tipos de pacientes que apresentavam alterações em mucosa oral, envolvendo doenças fúngicas, hiperplasias inflamatórias, estomatites e hiperemias.

Tabela 3. Apresentação de lesão em mucosa oral em diferentes pacientes submetidos a transplante hepático, cardíaco e renal no Conjunto Hospitalar de Sorocaba no período de 2002 a 2007.

\begin{tabular}{lllllll}
\hline & \multicolumn{2}{l}{ Feminino } & \multicolumn{4}{c}{ Masculino } \\
\hline Transplante & Com & Sem & \%total & Com & sem & $\%$ total \\
Rim & 3 & 19 & $14,28 \%$ & 2 & 19 & $9,52 \%$ \\
Coração & 0 & 8 & $0 \%$ & 1 & 7 & $12,50 \%$ \\
Figado & 19 & 35 & $35,18 \%$ & 19 & 91 & $17,27 \%$ \\
Total & 22 & 62 & & 22 & 117 &
\end{tabular}

Com base nos dados apresentados na tabela acima, observam-se que os pacientes submetidos ao transplante hepático apresentaram os maiores índices de alterações estomatológicas $(22,8 \%$ dos casos) quando comparados aos indivíduos que seriam submetidos ao transplante renal ( $11 \%$ dos casos) e transplante cardíaco $(6,25 \%$ dos casos). Em relação ao número de pacientes que apresentavam lesão na boca, o grupo do gênero masculino apresentou dados não analisáveis estatisticamente. O nível de significância dos resultados apresentados sugere que a amostra de pacientes que apresentavam lesão em boca entre os transplantados hepáticos do gênero feminino, foi maior do que as demais.

Em relação ao perfil bucal apresentado entre os grupos de pacientes listados para transplante de fígado, rim e coração, as entidades patológicas orais apresentadas foram especificadas nas Figuras 5, 6 e 7, sendo que um percentual significativo de pacientes apresentava infecções bucais prévias à terapia de imunossupressão.

Figura 5: Relação das condições bucais apresentadas por pacientes listados para transplante hepático.

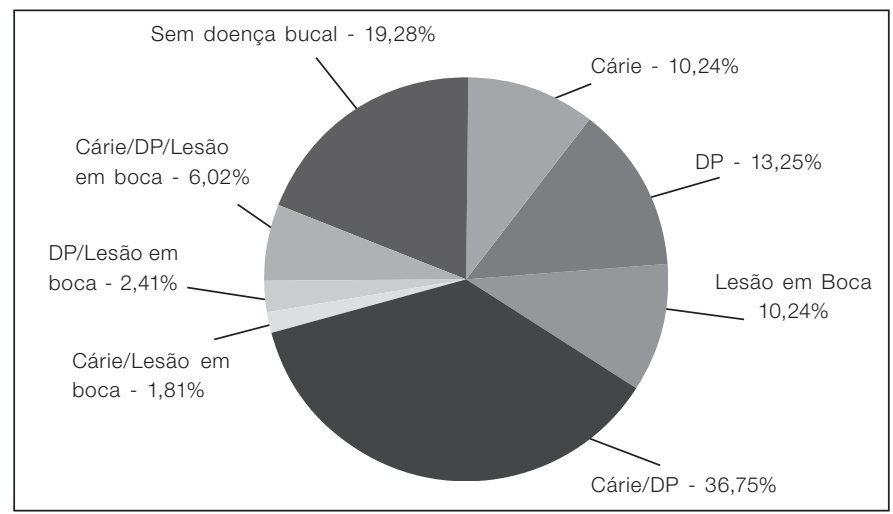

\section{DISCUSSÃO}

Com o aumento da incidência de pacientes submetidos a transplantes de órgãos sólidos, a presença de equipes de Odontologia pode e deve fazer parte da assistência a esses indivíduos. ${ }^{18} \mathrm{O}$ desequilíbrio entre a persistência de uma infecção bucal e a regulação imunológica do hospedeiro é significativa em muitas condições imunológicas que associam o transplante de órgãos sólidos. ${ }^{19}$

As infecções de origem bucal influenciam o sucesso do tratamento, se não forem diagnosticadas e eliminadas previamente ao período
Figura 6: Relação das condições bucais apresentadas por pacientes listados para transplante renal.

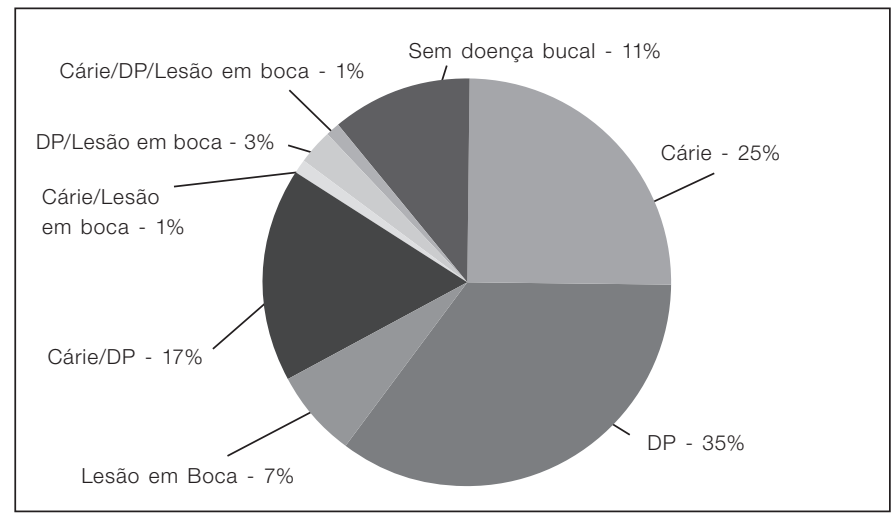

Figura 7: Relação das condições bucais apresentadas por pacientes listados para transplante cardíaco.

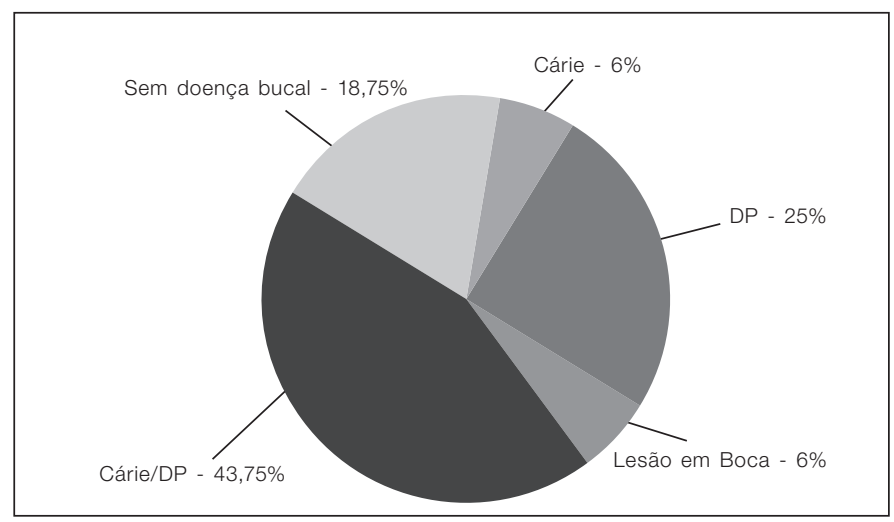

que envolve a imunossupressão, para que não haja rejeição do órgão implantado. ${ }^{20}$

Os dados apresentados neste estudo apontam uma documentação significativa de infecções odontogênicas em candidatos a transplante de órgãos sólidos, especificamente os submetidos ao transplante hepático, renal e cardíaco. Os focos odontogênicos em boca alojam quantidades consideráveis de bactérias periodontopatogênicas, as quais poderão comprometer a saúde sistêmica desses indivíduos, quando os estes apresentam comprometimento de suas defesas, ${ }^{21}$ afetando diretamente o sucesso do tratamento médico inicialmente proposto. ${ }^{1}$

A incidência de alterações estomatológicas foi elevada entre os grupos de indivíduos transplantados, com percentagens de 80,72\% em indivíduos que seriam submetidos ao transplante hepático, de $81,40 \%$ de pacientes com falência renal e de $81,25 \%$ de pacientes listados para transplante cardíaco.

Os índices de infecções na boca também foram significantes entre os grupos, sendo que o perfil das condições orais dos indivíduos submetidos ao transplante renal foi marcado pela presença de doença periodontal $(60,46 \%$ dos casos) enquanto que o perfil das condições bucais dos pacientes submetidos ao transplante hepático e cardíaco foram evidenciadas com a presença de doença cárie e doença periodontal concomitantes em $36,75 \%$ dos casos e de 43,75\% dos casos, respectivamente.

Em relação ao gênero, as mulheres que seriam submetidas ao transplante cardíaco foram as que apresentaram piores condições 
orais, com os maiores índices de doenças bucais quando comparados aos demais grupos, apresentando elevados percentuais de cárie $(75 \%)$ e de doença periodontal $(87,5 \%)$.

O ajuste das condições orais, com a remoção de focos odontogênicos antes do transplante, pode reduzir as complicações durante os procedimentos médicos necessários aos receptores de órgãos sólidos. ${ }^{19}$ As infecções em boca podem ser relevantes e interferir no sucesso terapêutico dos pacientes submetidos aos transplantes, comprometendo a sua qualidade de vida. ${ }^{18}$

A instrução de higiene oral é uma estratégia efetiva para a redução de microrganismos periodontopatogênicos, diminuindo a incidência de bacteremias e posteriores complicações durante o período de imunossupressão. ${ }^{3}$ A criação de programas de cuidados orais e a educação do paciente quanto à higiene bucal são componentes chaves para prevenir e reduzir os quadros de infecções orais, ${ }^{8}$ ressaltando a importância dos cirurgiões dentistas em equipes multiprofissionais. ${ }^{18}$

\section{CONCLUSÃO}

Os índices de infecções bucais presentes nos pacientes que são submetidos ao transplante hepático, renal e cardíaco são elevados, o que poderá comprometer o sucesso do tratamento médico proposto, devido à presença de complicações tardias. Esse fato ressalta a importância dos cirurgiões dentistas no acompanhamento prévio ao procedimento cirúrgico desses indivíduos, sendo componentes chaves na promoção de qualidade de vida desses pacientes.

\section{ABSTRACT}

Patients with chronic diseases scheduled for solid organ transplantation have systemic conditions that compromise their immune system that predisposes to a potential risk for infection, which should be prevented for the post-transplantation period, especially with the use of immunosuppressant. Infectious foci in the oral cavity after transplantation are a concern under such condition, requiring special attention. Purpose: To evaluate data related to the oral health condition of patients in a schedule for solid organ transplantation; this article emphasizes the importance of dental care ahead of the transplantation. Methods: This is a quantitative, descriptive, exploratory and retrospective study. It was analyzed 225 records of patients in liver, kidney and heart transplant schedule, the data related to oral conditions and possible infectious risks (cavities, periodontal disease, and infectious lesions on the mucosa membrane) attained upon the assessment of odontologic reports. Statistical methods were used to the analysis of significance by the chi-square and Fisher exact test with significance ( $\mathrm{p}<0.05$ ). Results: It was reported the presence of oral changes showing Infectious foci in (134/166) 80.72\% of patients in liver transplant program, (35/43) 81.39\% in renal transplant program, and (13/16) 81 25\% in heart transplant. Conclusion: Data showed that patients in patients in an organ transplant schedule, the incidence of oral Infectious foci is significant, suggesting that oral treatment ahead to the transplantation is important because of the immunosuppressant condition of patients in transplantation programs.

Keywords: Liver Transplantation, Kidney Transplantation, Heart Transplantation, Infection, Oral Health, Prevention \& Control.

\section{REFERÊNCIAS:}

1. Cunha CB, León ACP, Schramm JMA, Carvalho MS, Souza Júnior PRB, Chain R. Tempo até o transplante e sobrevida em pacientes com insuficiência renal crônica no Estado do Rio de Janeiro, Brasil, 1998-2002. Cadernos de Saúde Pública. 2007 Abr;23(4):43-7.

2. Harrison J, Mcmaster P. The role of orthotopic liver transplantation in the management of sclerosing cholangitis. Hepatology. 1994;(20 Suppl):14-9.

3. Martins FPP, Gonçalves RT, Fonseca LMB, Gutfilen B. Correlação do esquema de imunossupressão com complicações pós-operatórias em transplantes renais através do uso da cintilografia renal dinâmica. Radiol Brás. 2001 Set/Out; 34(5):165-74.

4. Costa JZ Filho, Padilha WSM, Santos EKN. Cuidados odontológicos em portadores de insuficiência renal crônica. Rev Cir Traumatol Buco-Maxilo-Fac. 2007 Abr/Jun; $7(2): 19-28$

5. Gill Y, Scully C. Orofacial odontogenic infections : review of microbiology and current treatment. Oral Med Oral Pathol. 1990;70:155-8.

6. Morhart RE, Fitzgerald RJ. Nutritional determinants of the ecology of the oral flora. Dent Clin North Am. 1976;20:473-89.

7. Oliveira FDF, Santos RL, Gusmão ES, Ramos CG, Silveira RCJ. Condição periodontal de pacientes transplantados renais que fazem uso da Ciclosporina-A (CsA). Odontologia Clín.-Científ. 2003 Maio/Ago;2(2):95-102.

8. Ruby J, Barbeau J. The bucale puzzle: the symbiotic nature of endogenous infections of the oral cavity. Can J Infect Dis. 2002;13(1):34-41.

9. Sassone LM. Microbiota de infecções endodônticas primárias e sua relação com aspectos clínicos radiográficos. Rio de Janeiro: [editor desconhecido]; 2005.150 p.

10. Souza CRD, SA, Guerra RNM, Monteiro S, Silveira EJD, Pereira ALA. Avaliação da condição periodontal de pacientes renais em hemodiálise. Rev Assoc Med Bras. 2005;51(5):285-9.

11. MouradG, Garrigue V, SquiffletJP, etal. Inductionversus noninduction in renal transplant recipients with tacrolimus-based immunosuppression. Transplantation. $2001 \mathrm{Sep}$ 27;72(6):1050-5.

12. Topazian TG, Goldenberg MH, Hupp JR. Princípios do tratamento cirúrgico e farmacológico de infecções. In: Peterson J. Infecções orofacias e maxilofaciais. 2006;4:415-6.

13. Williams R, Wendow J. Indications for orthotopic liver transplantation in fulminant liver failure. Hepatology. 1994;20(Suppl).

14. Noronha IL, Ferraz AS, Silva Filho AP, Saitovich D, Carvalho DBM, Paula FJ, et al. Transplante renal: indicações e contra-indicações. Sociedade Brasileira de Nefrologia Sociedade Brasileira de Urologia. 2006;2:54-62.

15. First MR. Tacrolimus based immunosuppression. J Nephrol. 2004 Nov-Dec;17 (Suppl 8):S25-31.

16. Pescovitz MD, Govani M. Sirolimus and mycophenolate mofetil for calcineurin-free immunosuppression in renal transplant recipients. Am J Kidney Dis. Oct 2001;38(4 Suppl 2):S16-21.

17. Kahan BD. Sirolimus-based immunosuppression: present state of the art. J Nephrol. 2004 Nov-Dec;17(Suppl 8):S32-9. 
18. Santos PSS, Bitu F, Coracin FL, Sobrinho RM, Lima RB. Complicações orais associadas aos transplante de órgãos e tecidos: revisão de literatura. JBT. 2009;12:1064-69.

19. Gulleç AT, Demirbilek M, Seçkin D, Can F, Saraya Y, Sarifakiogluu E, et al. Superficial fungical infections in 102 patients renal transplant recipients: a case- control study. J Am Acad Dermatol. 2003;49:187-92.

20. Rustemeyer J, Bremerich A. Necessity of surgical dental foci in treatment prior to organ transplantation and heart valve replacement. Clin Oral Invest. 2007;2(11):171-4.

21. Topazian RG, Goldemberg RH, Hupp JR. Infecções orais e maxilofaciais. 4.ed. [local desconhecido]: [editor desconhecido]; 2006. 